\title{
On the Fixation or Non-Fixation of Adaptive Inversions
}

\author{
Brian Charlesworth ${ }^{1}$ and Thomas Flatt ${ }^{2}$ \\ ${ }^{1}$ University of Edinburgh \\ ${ }^{2}$ Fribourg University
}

March 3, 2021

\begin{abstract}
Several recent publications have stated that epistatic fitness interactions cause the fixation of inversions that suppress recombination among the loci involved. Under this model, however, the suppression of recombination in an inversion heterozygote creates a form of heterozygote advantage, which prevents the inversion from becoming fixed by selection. This process has been explicitly modelled by previous workers.
\end{abstract}

\section{On the Fixation or Non-Fixation of Adaptive Inversions}

Brian Charlesworth ${ }^{1}$ and Thomas Flatt $^{2}$

${ }^{1}$ Institute of Evolutionary of Evolutionary Biology, School of Biological Sciences, University of Edinburgh, Edinburgh UK

${ }^{2}$ Department of Biology, University of Fribourg, Fribourg, Switzerland

Corresponding author: Brian Charlesworth

Email:Brian.Charlesworth@ed.ac.uk

Keywords: balanced polymorphism, epistasis, fitness, inversions, linkage disequilibrium recombination suppression

\section{Abstract}

Several recent publications have stated that epistatic fitness interactions cause the fixation of inversions that suppress recombination among the loci involved. Under this model, however, the suppression of recombination in an inversion heterozygote creates a form of heterozygote advantage, which prevents the inversion from becoming fixed by selection. This process has been explicitly modelled by previous workers.

There is a growing interest in the evolutionary role of inversions and other types of chromosome rearrangements, with several recent review papers (e.g., Wellenreuther \& Bernatchez, 2018; Faria et al., 2019; Kapun \& Flatt, 2019; Huang and Rieseberg, 2020) and a special issue ofMolecular Ecology devoted to this topic (Wellenreuther et al., 2019, and references therein). As these papers have pointed out, there are multiple ways in which natural selection can act on inversions. In order to discriminate between different hypotheses, it is necessary to have a clear understanding of their observable consequences. Unfortunately, there appears to be a serious misconception about one process that has been proposed as providing a selective advantage to an inversion. This involves Dobzhansky's (e.g., Dobzhansky, 1949, 1950, 1951) concept of "coadaptation" among polymorphic loci that interact in their effects on fitness, an idea that traces back to Fisher (1930, pp.102-104). 
In its simplest form, this model invokes two loci, $A$ and $B$, each segregating for a pair of alleles $\left(A_{1}\right.$ vs $A_{2}$, $\mathrm{B}_{1}$ vs $\mathrm{B}_{2}$ ) in a diploid, randomly mating population. If the fitness effects of the two loci are epistatic, in the sense that the fitnesses of the nine possible diploid genotypes at the two loci deviate from those predicted by additive combinations of the effects of the alleles at each locus (Fisher, 1918), it is possible for linkage disequilibrium (LD) to be maintained in the face of recombination at a stable equilibrium where both loci are polymorphic, such that the fitter combinations of alleles are in excess of the frequencies expected by randomly combining alleles according to their frequencies (Fisher, 1930; Kimura, 1956; Lewontin \& Kojima, 1960; Karlin, 1975). There is then selection for modifiers that reduce the rate of recombination between the two loci (Fisher, 1930; Kimura, 1956; Feldman, 1972). The same principle applies to more general multilocus systems in randomly mating populations (Charlesworth, 1976; Zhivotovsky et al., 1994), but not necessarily to partially inbreeding populations (Charlesworth et al., 1979). In particular, an inversion that arises on a haplotype that is present in excess of random expectation (and is thus fitter than average) experiences a selective advantage if recombination is suppressed in heterozygotes for the inversion, simply because the inversion maintains its association with higher than average fitness (Kimura, 1956; Charlesworth \& Charlesworth, 1973).

The misconception is that this process causes the inversion to spread to fixation, implying that it cannot explain the balanced inversion polymorphisms that have been the subject of so much recent attention. This claim appears to have originated in Table 1 of Kirkpatrick \& Barton (2006), and has been repeated in review papers by Hoffmann \& Rieseberg (2008), Kapun \& Flatt (2019), and Huang \& Rieseberg (2020). However, it overlooks the fact that crossing over is suppressed only in inversion heterozygotes, so that the selective benefits of recombination suppression confer a heterozygote advantage to the inversion, and hence prevent its fixation. This scenario was first modelled by Kimura (1956), and examined in more detail by Charlesworth (1974), who showed that the exchange of alleles among inverted and standard arrangements by gene conversion or double crossing over does not prevent the establishment and maintenance of an inversion polymorphism when there is epistatic selection. An example of this mechanism for maintaining inversion polymorphisms is provided by the epistatic interactions among the different components of segregation distorter systems, and probably explains the frequent association of such systems with inversions (Fuller et al., 2020). Direct effects of inversions in causing segregation distortion should, therefore, not be assumed.

\section{References}

Charlesworth, B. (1974). Inversion polymorphism in a two-locus genetic system. Genetical Research , 23, 259-280. https://doi.org/10.1017/S0016672300014919

Charlesworth, B. (1976). Recombination modification in a fluctuating environment. Genetics 83:181-195. https://doi.org/10.1093/genetics/83.1.181

Charlesworth D, Charlesworth B \& Strobeck C. 1979. Selection for recombination in partially self-fertilizing species. Genetics93:237-244. https://doi.org/10.1093/genetics/93.1.237

Dobzhansky, T. (1949). Observations and experiments on natural selection in Drosophila . Proceedings of the 8th International Congress of Genetics, republished in Hereditas, 35 , 210-224.

Dobzhansky, T. (1950). Genetics of natural populations. XIX. Origin of heterosis through natural selection in populations of Drosophila pseudoobscura . Genetics , 35 , 288-302. https://doi.org/10.1093/genetics/35.3.288

Dobzhansky, T. (1951). Genetics and the Origin of Species, 3rd edition. New York, NY: Columbia University Press.

Faria, R., Johannesson, K., Butlin, R.K., \& Westram, A.M. (2019). Evolving inversions. Trends in Ecology and Evolution, 34 , 239-248. https://doi.org/10.1016/j.tree.2018.12.005

Feldman, M.W. (1972). Selection for linkage modification: I. Random mating populations. Theoretical Population Biology , 3 , 324-346. https://doi.org/10.1016/0040-5809(72)90007-X 
Fisher, R.A. (1918). The correlation between relatives on the supposition of Mendelian inheritance. Transactions of the Royal Society of Edinburgh , 52 , 399-433. https://doi.org/10.1017/S0080456800012163

Fisher, R.A. (1930). The Genetical Theory of Natural Selection . Oxford, UK: Clarendon Press.

Fuller, Z.L., Koury, S.A., Leonard, C.J., Young, R.E., Ikegami, K., Westlake, J., Richards, S., Schaeffer, S.W., \& Phadnis, N. (2020). Extensive recombination suppression and epistatic selection causes chromosomewide differentiation of a selfish sex chromosome inDrosophila pseudoobscura. Genetics , 216, 205-226. https://doi.org/10.1534/genetics.120.303460

Hoffmann, A.A., \& Rieseberg, L.H. (2008). Revisiting the impact of inversions in evolution: from population genetic markers to drivers of adaptive shifts and speciation? Annual Review of Ecology, Evolution, and Systematics , 39 , 21-42. https://doi.org/10.1146/annurev.ecolsys.39.110707.173532

Huang, K., \& Rieseberg, L.H. (2020). Frequency, origins, and evolutionary role of chromosomal inversions in plants. Frontiers in Plant Science, 1 , 296. https://doi.org/10.3389/fpls.2020.00296

Kapun, M., \& Flatt, T. (2019). The adaptive significance of chromosomal inversion polymorphisms in Drosophila melanogaster .Molecular Ecology , 28 , 1263-1282. https://doi.org/10.1111/mec.14871

Karlin, S. (1975). General two-locus selection models: Some objectives, results and interpretations. Theoretical Population Biology ,7 , 364-398. https://doi.org/10.1016/0040-5809(75)90025-8

Kimura, M. (1956). A model of a genetic system which leads to closer linkage by natural selection. Evolution , 10 , 278-287. https://doi.org/10.1111/j.1558-5646.1956.tb02852.x

Kirkpatrick, M., \& Barton, N. (2006). Chromosome inversions, local adaptation and speciation. Genetics , 173 , 419-434. https://doi.org/10.1534/genetics.105.047985

Lewontin, R.C., \& Kojima, K-i. (1960). The evolutionary dynamics of complex polymorphisms. Evolution , 14 , 458-472. https://doi.org/10.1111/j.1558-5646.1960.tb03113.x

Wellenreuther, M., \& Bernatchez, L. (2018). Eco-evolutionary genomics of chromosomal inversions. Trends in Ecology and Evolution ,33 , 427-440.https://doi.org/10.1016/j.tree.2018.04.002

Wellenreuther, M., Mérot, C., Berdan, E., Bernatchez, L. (2019). Going beyond SNPs: The role of structural genomic variants in adaptive evolution and species diversification. Molecular Ecology ,28 , 1203-1209. https://doi.org/10.1111/mec.15066

Zhivotovsky, L.A., Feldman, M.W., Christiansen, F.B. (1994). Evolution of recombination among multiple selected loci: a generalized reduction principle. Proceedings of the National Academy of Sciences USA ,91, 1079-1083. https://doi.org/10.1073/pnas.91.3.1079

Data accessibility: No new data were generated in this study.

Author contributions: Both authors wrote the paper. 